\title{
ANÁLISIS DE LA INTERLENGUA DE ITALIANOS APRENDICES DE ESPAÑOL
}

\author{
Esther Gutiérrez Quintana \\ Instituto Cervantes de Varsovia - Editorial SGEL \\ esthergutierrezquintana@hotmail.com
}

\begin{abstract}
Resumen
¿Cuál es el grado de influencia de la lengua materna en el aprendizaje del español por italianos?, ¿existen errores característicos de estos estudiantes?, ¿utilizan estrategias de aprendizaje similares? Éstas son sólo algunas cuestiones que queremos plasmar en el desarrollo de este trabajo. Comenzamos este artículo presentando una breve revisión de los principales modelos de investigación en Lingüistica Contrastiva y valoramos su relevancia actual. Exponemos los resultados de un trabajo empírico en el que hemos analizado un corpus formado por textos escritos por tres grupos de estudiantes italianos de español de la Università degli Studi di Sassari (Italia). Hemos identifcado, descrito y clasificado los errores más frecuentes con objeto de determinar las áreas que presentan mayor dificultad para su asimilación y la evolución de dichos errores en diferentes niveles de competencia lingüística. Hemos localizado los errores en su contexto, los hemos clasificado en categorías lingüísticas y los hemos descrito exhaustivamente determinando sus causas, frecuencia, gravedad y evolución por medio de unos criterios de análisis previamente establecidos. Los resultados han sido sistematizados tanto desde el punto de vista cuantitativo como cualitativo.

PALABRAS CLAVE: Interlengua, lengua materna, análisis de errores áreas dificultosas, italófonos.
\end{abstract}

\begin{abstract}
To what extent does the mother tongue influence the learning process of Italian students of Spanish? Do these students make typical mistakes? Do they use similar learning strategies? These are just some of the issues addressed in this work. The article begins with a brief review of the main models of investigation used in Contrastive Linguistics and the evaluation of their present relevance. It continues with the presentation of the results of an empirical project, in which a body of texts written by three groups of Italian students of Spanish at the Università degli Studi in Sassari, Italy, were analyzed. The most frequent mistakes have been identified, described and classified, to determine which areas present greater difficulties of assimilation, and their evolution at various levels of linguistic competence, has also been analysed. The mistakes have been placed within their context, classified into linguistic categories and described exhaustively, identifying their cause, frequency, seriousness and evolution, by using a previously established criteria of analysis. The results are grouped according to quantity and quality.
\end{abstract}

KEY WORDS: Interlenguage, Maternal language, Analysis of errors, Difficult areas, Italian informants.

\section{Introducción}

Presentamos en ese artículo los resultados de un trabajo empírico en el que hemos analizado un corpus de textos escritos de tres grupos de estudiantes italianos de español de la Università degli Studi di Sassari (Italia). Hemos identificado, descrito y clasificado los errores más frecuentes con objeto de determinar las áreas que presentan mayor dificultad para su asimilación y la evolución de dichos errores en diferentes niveles de competencia lingüística. Hemos localizado los errores en su contexto, los hemos clasificado en catego- 
rías lingüísticas y los hemos descrito exhaustivamente determinando sus causas, frecuencia, gravedad y evolución por medio de unos criterios de análisis previamente establecidos. Los resultados han sido sistematizados tanto desde el punto de vista cuantitativo como cualitativo'.

Son dos los motivos que nos llevaron a planteamos la realización de un trabajo empírico de este tipo, en primer lugar, la necesidad de averiguar cómo perciben los estudiantes italianos su propio aprendizaje del español y, en segundo lugar, la posibilidad de indagar sobre algunos fenómenos intuidos durante la actividad didáctica como, por ejemplo, la recurrencia constante de un mismo tipo de error en grupos de diferente nivel y especialidad académica. Ante una situación de este tipo, en la que salta a la vista la repetición de ciertas formas idiosincrásicas es fácil cuestionarse las causas de dicho fenómeno y es fácil también llegar a la conclusión de que el origen de dichos errores podría ser el mismo y, por tanto, que determinando sus causas se pueden solventar muchos problemas de enseñanza. Para ello, era imprescindible establecer una metodología válida, aplicable a otros contextos de enseñanza y obtener unos resultados lo suficientemente representativos para evitar muchos casos de fosilización de errores y, sobre todo, que hiciesen progresar nuestra práctica docente, volviéndose más fructífera ${ }^{2}$. Pero examinando la interlengua de un grupo de italianos aprendices de español son muchas más las cuestiones que podemos esclarecer, entre otras, se puede comprobar si existen diferentes etapas perceptibles en el proceso de adquisición del español por parte de grupos homogéneos, cuáles son las principales dificultades que encuentran los estudiantes en cada uno de estos niveles y qué mecanismos se activan para solventar esos problemas. Es decir, además de identificar cuáles son las áreas lingüísticas más inestables y cuáles presentan mayor riesgo de fosilización, hemos analizado como evolucionan los errores en las varias etapas de aprendizaje, cuáles permanecen y cuáles desaparecen cuando el estudiante avanza en su aprendizaje de la lengua. El objetivo principal es, por tanto, acercarnos a ese momento de competencia transitoria tan delicado como es la interlengua de los estudiantes italianos y entender una serie de peculiaridades de la misma en distintas etapas de su aprendizaje ${ }^{3}$.

Es justo señalar que hemos tenido que afrontar ciertas dificultades a la hora de llevar a cabo esta investigación. Uno de los mayores problemas que surgieron durante el planteamiento de los objetivos y de la recogida del corpus fue la posibilidad más o menos real de generalizar los resultados obtenidos, pues sabemos que cada situación de enseñanza presenta unas determinadas particularidades motivadas, principalmente, por las variantes específicas del grupo y del contexto de enseñanza. Por eso, somos conscientes de que los resultados

\footnotetext{
1 Ver Gutiérrez Quintana, E. (2004c): “Un posible modelo de análisis contrastivo entre lenguas afines". En Actas del I Simposio de Didáctica del Español para Extranjeros. Teoria y Práctica, Instituto Cervantes de Rio de Janeiro y Gutiérrez Quintana, E. (2004d): Aspectos gramaticales contrastivos en español e italiano. Alcalá de Henares, Tesis doctoral defendida en la Universidad de Alcalá.

2 Hemos estructurado este trabajo siguiendo las pautas propuestas por Larsen-Freeman, D. y M. H. Long (1994) (versión española de Molina Martos, I. y P. Benítez Pérez): Introducción al estudio de la adquisición de segundas lenguas. Madrid, Gredos manuales.

3 Es importante también señalar que este tipo de investigaciones deben tener un carácter fundamentalmente práctico, la finalidad principal deber ser, no sólo obtener una visión panorámica que nos permita comprender mejor el proceso de enseñanza-aprendizaje de segundas lenguas, sino también dirigir los esfuerzos hacia una posible y futura aplicación didáctica que permita la superación de los errores, por medio, por ejemplo, de la creación de actividades de refuerzo y de la utilización de técnicas didácticas específicas y adecuadas.
} 
de este estudio reflejan con claridad las características de la interlengua de un determinado grupo homogéneo pero será difícilmente aplicable en su totalidad a cualquier situación de enseñanza del español a italianos. Otra dificultad considerable, en este caso de tipo puramente metodológico, fue establecer un juicio de gramaticalidad en el que basarnos a la hora de detectar y analizar las producciones idiosincrásicas. No olvidemos que la norma lingüística es, en un cierto sentido, un concepto subjetivo, pues la competencia como hablante nativo varía de persona a persona y para establecer una norma lingüística válida a la hora de realizar un trabajo empírico de Análisis de Errores, es necesario que el investigador sea crítico en la selección de los contenidos gramaticales, es decir, debe tener una sólida base teórica pero debe también apoyarse en su propia experiencia como nativo, proponiendo una norma única y prestando atención a las desviaciones causadas por la realidad de uso de los varios elementos gramaticales. Y, por último, un tercer problema que tuvimos que afrontar fue el hecho de compartir y conocer la lengua nativa de los estudiantes que, aunque a priori es, sin duda, una ventaja, en ocasiones conlleva ciertos peligros, sobre todo si el profesor se acostumbra a un cierto tipo de error modificando su criterio sobre la gravedad del mismo o llevándole a dudar sobre su propio sistema lingüístico.

Para elaborar este trabajo nos hemos apoyado en los preceptos del Análisis de Errores (pero adoptando también la metodología propia del modelo de Análisis Contrastivo), cuyo propósito es la explicación a través del error de los mecanismos de adquisición de segundas lenguas, así como la determinación de la mayor o menor influencia de la lengua materna como factor determinante en la persistencia de determinados errores.

Desde el punto de vista bibliográfico, hemos consultado gramáticas del español y del italiano ${ }^{4}$, textos teóricos sobre gramática española ${ }^{5}$, diccionarios bilingües ${ }^{6}$, manuales de lingüística ${ }^{7}$, artículos específicos de corte contrastivo sobre sectores concretos de la lengua ${ }^{8}$ y otros materiales.

\section{Aspectos teóricos}

Presentamos un breve recorrido por los diferentes modelos de investigación en Lingüística Contrastiva, es decir, el Análisis Contrastivo, el Análisis de Errores y el modelo de Interlengua, así como su estado actual, sus hipótesis de trabajo, la mayor o menor repercusión y aportaciones al panorama de la enseñanza de lenguas extranjeras y las críticas que estos métodos han recibido. Los tres modelos presentan diversas áreas en común, puesto que todos ellos miran hacia la consecución de un objetivo único: el estudio del proceso de aprendizaje de una segunda lengua y, en concreto, de la lengua de los estudiantes como sistema de comunicación. Pero presentan también puntos de contraste, por lo que presentamos cada

\footnotetext{
4 Alarcos Llorach: 1995, Carrera Díaz: 2002, Gómez Torrego: 1998, Matte Bon: 2001, Battaglia: 1989. Bellini: 1994, Finasi Parolo y Giovannetti Muñoz: 1998.

5 Álvarez Martínez: 1989, Fernández: 1999.

6 Calvi y Monti: 1991, Calvo Rigual y Giordano: 1997, Martínez Amador: 1988, Sañé: 1992, Tam: 1993 y Aragona: 1992.

7 Alvar Ezquerra (dir.): 2000, Alcaraz Varó y Martínez Linares: 1997, Ciliberti: 1994

8 Betti: 2002, Bini y Guil: 2002, Caballero Rubio y Corral Hernández: 1997, Castañeda Castro y Peña Sánchez: 1995, De Kock: 1996, Gil: 1994, González Royo: 1986, Gutiérrez Quintana: 2004a, 2004b, 2004d, 2005a, 2005b, López 
modelo separadamente, serán diferentes, por ejemplo, el corpus de datos utilizado, los fines didácticos que se persiguen y los principios metodológicos en los que se basan?.

Desde sus inicios, la Lingüística Aplicada ha sido una disciplina científica de gran relevancia, se apoya en los conocimientos teóricos que ofrece la Lingüística Teórica y su objetivo principal es la resolución de problemas lingüísticos generados por el uso del lenguaje en una comunidad. Se caracteriza también por la evolución que como disciplina ha experimentado a lo largo del tiempo, abarcando, especialmente durante los años cuarenta, el análisis del proceso de enseñanza de la lengua materna para concentrarse posteriormente en lo concerniente al proceso de aprendizaje de lenguas segundas ${ }^{10}$. En el ámbito de la Lingüística Aplicada nació una nueva disciplina, la Lingüística Contrastiva, que cuenta con una gran tradición en Estados Unidos, en la Europa Central y en la antigua Europa del Este. El término se acuñó en 1940 y ya en 1946 fue reconocida como disciplina científica independiente por la Universidad de Michigan, sobre todo gracias al impulso y difusión que suscitó la aparición de diversas revistas y asociaciones que difundieron estas nuevas perspectivas teórico-prácticas ${ }^{11}$. Al igual que a muchos otros investigadores que llevan a cabo su propia labor con grupos homogéneos y, aún con más razón en el ámbito de lenguas afines al español, nos interesa especialmente el campo de acción de la Lingüística Contrastiva puesto que estudia concretamente el contraste entre dos o más sistemas lingüísticos. Estas comparaciones se llevan a cabo de manera sincrónica -en el ámbito de estudio de los universales lingüísticos- o bien pueden tener como objeto la aportación de nuevos datos sobre los efectos que tiene una lengua sobre otra en el aprendizaje de lenguas segundas.

El primer modelo de investigación que surgió, en el ámbito de la Lingüística Contrastiva, fue el Análisis Contrastivo ${ }^{12}$. Inaugurado en $1945^{13}$ con el trabajo Teaching and Learning English as a Foreign Language publicado por Fries, este modelo alcanza su auge en 1957 con Linguistics across Cultures, de Robert Lado ${ }^{14}$. Se fundamenta en la creencia de que el aprendizaje de una segunda lengua es la formación de nuevos hábitos lingüísticos gracias a la labor facilitadota de un hábito viejo, es decir, el conocimiento que el estudiante posee de su lengua nativa y dependiendo de las similitudes y diferencias entre los viejos hábitos y los nuevos este proceso será más o menos productivo. Según esta concepción, se tendrá en cuenta la distancia interlingüistica, es decir, la distancia que media entre la lengua nativa

9 Referencias fundamentales en el ámbito español para trazar una panorámica clara de los varios modelos en Lingüística Contrastiva son Baralo Otonello: 1994, 1999, Santos Gargallo: 1993, Fernández López: 1997 y Liceras: 1992.

10 Lo resume muy bien Isabel Santos Gargallo en Santos Gargallo, I. (1999): Lingüistica Aplicada en la enseñanza-aprendizaje del español como lengua extranjera. Madrid, Arco/Libros.

11 En 1948 apareció la revista Language Learning (LL), subtitulada Journal of Applied Linguistics; en 1964 se creó la Association Internationale de Linguistique Appliquée y, por último, en 1968 se publicó el primer número de la International Review of Applied Linguistics (IRAL).

12 Un artículo que resume muy bien este modelo es Wardhaugh, R. (1970): “The Contrastive Analysis Hypothesis", TESOL Quartely 4, págs. 123-136.

13 Fries, C. (1945): Teaching and Learning English as a Foreign Language. Ann Arbor, University of Michigan Press.

14 Lado, R. (1957): Linguistics Across Cultures. Ann Arbr, University of Michigan Press. El objetivo de este trabajo era identificar las áreas problemáticas en el proceso de aprendizaje de una segunda lengua y, en concreto, los elementos de la lengua materna del alumno que, por interferencia, dificultan el proceso de aprendizaje y son fuente directa de error, en definitiva, compara ambos sistemas lingüísticos para llegar a elaborar predicciones sobre las áreas de dificultad y extraer resultados que sirvan para preparar nuevos materiales didácticos. 
y la lengua meta, de modo que cuanto mayor sea la diferencia entre ambas lenguas más dificultoso resultará el aprendizaje y, en ese caso, también aumentará la posibilidad de interferencia, o lo que es lo mismo, de utilización por parte del alumno en la lengua meta de un rasgo fonético, morfológico, sintáctico o léxico tomado de su lengua nativa.

Fue en los años setenta cuando el Análisis Contrastivo comenzó a dejar paso al Análisis de Errores, ya que se observó que muchos errores no eran fruto de la interferencia de la lengua materna, al igual que se puso de manifiesto la insuficiencia descriptiva del modelo, pues analizaba superficialmente las diferencias cuantitativas entre los sistemas lingüísticos en detrimento de las descripciones de carácter cualitativo. El Análisis de Errores propone un replanteamiento tanto de la teoría del aprendizaje como del tratamiento de los errores, revalorizando el propio concepto y ampliando las posibles fuentes causantes del error. Es muy indicativa la evolución que el concepto de error experimentó en este período, pasó a ser visto positivamente, como una señal clara de que el proceso de aprendizaje estaba teniendo lugar y de que el estudiante atravesaba una de las etapas o estados de dicho aprendizaje. Fue S. Pit Corder en 1967 y $1982^{15}$ quien primero revalorizó el concepto de error como principal fuente de información sobre el proceso de aprendizaje de una $\mathrm{L} 2^{16}$. Se empezaron a estudiar los errores producidos por estudiantes de L2 con el objetivo, en un primer momento, de predecir y explicar tipologías de errores basadas en taxonomías gramaticales, pero después los esfuerzos se concentraron en el estudio de los efectos pragmáticos que los errores provocaban en el oyente y la valoración del efecto comunicativo de los mismos con el propósito de elaborar un programa de enseñanza-aprendizaje más efectivo. El Análisis de Errores es un modelo de gran utilidad para identificar y clasificar determinados errores en las producciones reales de los aprendices de lenguas segundas y para explicar tanto las fuentes como la gravedad de dicho error y buscar una terapia adecuada para superarlos.

En cuanto a la Hipótesis de la Interlengua ${ }^{17}$, se puede considerar la etapa sucesiva tras los modelos anteriores en el campo de la investigación científica orientada al estudio del proceso de aprendizaje de una L2 y su finalidad es el estudio de la producción total del estudiante de L2 para llegar a definir ese sistema. Frente a las disciplinas precedentes, sus estudios se aproximan más a la lengua producida por el estudiante de L2, se considera como un sistema lingüístico autónomo, un sistema de comunicación cuya complejidad aumenta mediante el proceso creativo que consiste en el aprendizaje por parte del alumno de nuevas estructuras y vocabulario. El proceso de aprendizaje de una segunda lengua se concibe como un conjunto de estructuras psicológicas subyacentes en la mente del aprendiz que se activan cuando se aprende una L2 y la interlengua como la progresión de los diversos estadios o

15 Corder, S. P. (1967): “The Significance of Learners Errors". En International Review of Applied Linguistics, vol. V, 4 y Corder, S. P. (1981): Error Analisis and Interlenguage. Oxford, University Press. Actualmente, otros autores han seguido la misma línea, ejemplos claros son los artículos "Hacia una valoración positiva del concepto de error" de Graciela Vázquez, "El error en el proceso de aprendizaje" de Blanco Picado, "Corregir y evaluar desde la perspectiva comunicativa" de Sonsoles Fernández, "El concepto de error: estado de la cuestión y posibles investigaciones" de Graciela Vázquez y el libro de De La Torre Aprender de los errores. El tratamiento didáctico de los errores como estrategia de innovación.

16 El artículo "The Significance of Learners Errors" supuso un cambio en su valoración, al menos en el plano teórico, exponiendo la triple utilidad de los errores, para el profesor, para el investigador y, aún más importante, para el que aprende.

17 Fue Selinker quien formuló en 1972 la Hipótesis de la Interlengua. Se puede consultar al respecto Selinker, L. (1992): Rediscovering Interlanguage, Londres, Longman. 
etapas por las que pasa el alumno durante el proceso de adquisición de una lengua que conforman un sistema lingüístico autónomo y diferente, tanto de la lengua materna como de la L2, y que puede describirse mediante un subconjunto de las reglas de la gramática de la lengua meta.

\section{Estado de la cuestión}

Como hemos visto, un acontecimiento primordial en el ámbito de la investigación en glosodidáctica de segundas lenguas es la llegada en los años setenta de la propuesta del Análisis de Errores, que suponía un mayor énfasis en la explicación a través del error de los mecanismos de adquisición de la L2. Más tarde, sobre todo en los años ochenta, tuvo lugar una recuperación de los estudios del Análisis Contrastivo, pero visto desde una nueva óptica. En primer lugar, se pone de manifiesto la influencia de la lengua materna como factor determinante no sólo en la persistencia de determinados errores, sino también en la omisión de ciertas estructuras por su diferencia interlingüística. En segundo lugar, se plantean nuevas condiciones que pueden favorecer la transferencia como los factores sociales, la distancia interlingüística, el nivel del aprendiz y el tipo de tarea que se realiza en el aula. En los años ochenta, es muy interesante también la consideración de la teoría de los universales lingüísticos, que parte de las teorías chomskianas y que considera el papel de la L1 y su relación con la Gramática Universal ${ }^{18}$, así como la influencia de la lengua materna en el proceso de adquisición de una segunda lengua. En los años noventa se llevaron a cabo estudios en, al menos, tres ámbitos diferentes: trabajos de naturaleza contrastiva con implicaciones didácticas, estudios de análisis de la interlengua empleada por el estudiante de L2 y estudios teóricos que establecen un vínculo entre la teoría lingüística y la teoría de la adquisición de L2. Actualmente, los estudios de Lingüística Contrastiva no son muy numerosos, pero el número de trabajos y publicaciones cuyo objeto de investigación es la enseñanza del español aumenta progresivamente, en primer lugar, por la creciente expansión de nuestra lengua y, en segundo lugar, porque es indudable que el Análisis Contrastivo basado en la descripción y contraste de lenguas es una gran ayuda para comprender y explicar el proceso de adquisición de una segunda lengua, así como un importante instrumento pedagógico que ayuda a comprender las causas de los errores cometidos por los estudiantes y los efectos de la lengua materna.

En cuanto a la situación actual de los estudios de tipo contrastivo entre español e italiano o de análisis de errores de italianos aprendices de español y viceversa, no sólo encontramos ya en el panorama bibliográfico español numerosos trabajos centrados en el análisis de la interlengua, sino que es además evidente el creciente número de congresos específicos de Lingüística Contrastiva (como los International Contrastive Linguistics Conference) en los que aparecen cada vez más trabajos que ponen en relación el español y el italiano (Gutiérrez Quintana: 2005b), así como las actas de congresos anuales de asociaciones orientadas esencialmente hacia la Lingüística Aplicada y a la enseñanza de lenguas como ASELE y AESLA (Fernández López: 1994, Galán Bobadilla: 1996, Luque Toro: 1994, Santos Gargallo: 1994,

18 Ver Adjémian, C. y J. M. Liceras (1984): "Accounting for Adult Acquisition of Relative Clauses: Universal Grammar, LI and Structuring the Intake". En Eckman, F., L. Bell y D. Nelson (eds.): Universals of Second Lanyuage Acquisition, Rowley, Mass., Newbury House. 
etc.) Otros materiales bibliográficos son las memorias de los Master de formación de profesores en E/LE (Penadés Martínez (coord.): 1999), monografias (Arribas y Landone: 2003), manuales (Gorris Yagüe: 2003), revistas especializadas (Matte Bon: 2004, Calvi: 2004) y publicaciones colectivas centrados en cuestiones específicas de Lingüística Contrastiva; es suficiente observar la creciente aparición en el mercado de manuales basados en el modelo de Análisis de Errores, como son los recientemente publicados en editoriales como Edelsa y Arco/Libros ${ }^{19}$.

\section{Metodología}

Uno de los pilares que sustentan teóricamente este trabajo son los criterios de clasificación de errores, que hemos determinado en una fase previa al trabajo de análisis de las producciones erróneas. Aunque somos conscientes de que la situación ideal es poder combinar varios planteamientos metodológicos, tras reflexionar sobre los objetivos específicos de nuestro trabajo, hemos optado por apoyarnos en la propuesta que, con las debidas adaptaciones, más se ajustaba a nuestras necesidades, es decir, la clasificación formulada por G. Vázquez (1991: 31 y 1998: 28). El primero de los cuatro criterios establecidos es el lingüistico, que se basa en la comparación del dialecto idiosincrásico —o producción del hablante no nativo en un momento determinado-, con la versión o producción que realizaria un hablante nativo ideal. Son cuatro las operaciones que hemos incluido bajo el criterio lingüístico: la adición, que consiste en agregar morfemas o palabras innecesarias en un contexto determinado, la omisión, proceso que consiste en la ausencia por supresión de morfemas y palabras no redundantes, la selección falsa, o elección incorrecta de palabras o vocablos en un contexto determinado y, por último, la colocación falsa, que consiste en colocar los elementos de una frase en un orden sintagmático incorrecto o inusual. El segundo criterio de clasificación es el criterio etiológico que, como su propio nombre indica, sirve para identificar las causas del error prestando atención a dos estrategias universales de aprendizaje: la transferencia y la interferencia; la primera se puede definir como el impacto de un sistema sobre otro cuyo resultado es la interlengua con su sistema de reglas propias y la interferencia, en cambio, es la estrategia que provoca errores o formas diferentes de las normas comúnmente aceptadas como correctas, clasificables en interlinguales -si se producen por la influencia de la L1 o de otra L3 - e intralinguales - provocados por el conflicto interno de las reglas de la propia L2-. Desde el punto de vista del criterio comunicativo, la clasificación se realiza dependiendo del efecto que producen en la transmisión del mensaje desde la perspectiva del oyente. Y, por último, con el criterio pedagógico se determinan los errores que caracterizan cada etapa del aprendizaje y se diferencian los errores transitorios de los errores sistemáticos, los primeros aparecen en determinadas etapas del aprendizaje y son errores de actuación fácilmente reparables, frente a los segundos, que reflejan un conocimiento deficiente de las reglas. Independientemente de la edad o nivel de instrucción del estudiante podrán darse casos de fosilización como consecuencia de circunstancias psicológicas especiales como la falta de atención, la concentración excesiva sobre el contenido en detrimento de la forma, o el cansancio, entre otras, y se puede definir como 
la tendencia a la reaparición inesperada de algunos errores que se creían ya superados y eliminados. Por último, los errores individuales son aquellos que aparecen en la interlengua de un hablante no nativo de manera particular frente a los errores colectivos que caracterizan la lengua de un grupo homogéneo o bien son errores de determinados aspectos de la L2 que se repiten y que mantienen una mayor tendencia a la fosilización.

El corpus sirvió de base para el estudio empírico está formado por un sólo tipo de prueba, una narración, puesto que permite a los informantes un alto grado de libertad y es un formato fácilmente adaptable a las exigencias de cada uno de ellos. De todos los procedimientos que existen para recoger datos, la redacción libre es quizás el menos controlado, ya que el investigador participa en su desarrollo sólo con la decisión del tema. Se intentó que la producción de los textos no estuviese condicionada - por eso los informantes no sabían qué elementos serían después analizados - y que la prueba fuera factible en términos de espacio, duración, etc. Es interesante aludir de nuevo a la posibilidad, en un futuro próximo, de ampliar este corpus de textos, compendiando además otros materiales de tipo oral, pues consideramos sugestiva la idea de comprobar si estos errores localizados reaparecen en muestras de lengua hablada.

Los textos versan sobre un tema previamente elegido por el alumno entre tres posibilidades. Para la elección de los temas tuvimos en cuenta varios requisitos: que fuesen cercanos a la realidad de los alumnos para obtener una producción espontánea y centrada en el contenido, que fueran temas más o menos personales de manera que, el hecho de hablar de sí mismos o de algo que conocían perfectamente, sirviese como fuente de motivación, que los argumentos propuestos no frenasen su creatividad por ser de dificil exposición y, por último, que la redacción resultase lo más abierta posible, evitando imposiciones y normas excesivas. Los alumnos no disponían de material de consulta, de manera que emplearon sus propias estrategias para la superación de las dificultades lingüísticas. Para realizar las pruebas dispusimos de dos horas de clase para cada grupo durante un arco temporal de cinco días en el propio entorno académico de los alumnos.

En la investigación participaron 44 estudiantes, 33 pertenecientes al segundo curso de español (el $73 \%$ ), 5 al tercero (el 11\%) y 7 al cuarto (el 16\%), todos ellos alumnos de los cursos impartidos por el Centro Linguistico di Ateneo de la Università degli Studi di Sassari y, en concreto, de la carrera universitaria Lingue e Letterature Straniere, especializada en el estudio minucioso de varias lenguas extranjeras. Era un grupo homogéneo, de nacionalidad y lengua materna italianas y con la particularidad de que todos los informantes se encontraban en una situación natural de bilingüismo ${ }^{20}$. En el momento de la compilación del corpus sus edades estaban comprendidas entre los veinte y los veintisiete años, con un total de diez hombres frente a cuarenta y tres mujeres, lo que suponía una inmensa mayoría de informantes femeninos. Los textos se recogieron durante el curso académico 2000/2001,

20 En Cerdeña ha persistido el uso generalizado, aunque de forma casi exclusivamente oral y siempre dependiendo de la situación de comunicación, del sardo o lengua sarda, que coexiste con el italiano. Esta particularidad es fundamental, ya que, aunque nos referimos sobre todo al italiano como lengua materna de los informantes, hemos comprobado que muchos de los errores encontrados, sobre todo de tipo léxico, proceden de la interferencia con el sardo. En este sentido, no olvidemos que se trata de una lengua neolatina que ha mantenido muchos rasgos arcaizantes por su difusión netamente oral y falta de normalización y que presenta muchos parecidos con el español, caracteristica que favorece la interferencia. 
concretamente a finales del mes de noviembre, de manera que los alumnos pertenecientes al segundo curso habían completado sólo un año de lengua española y los alumnos de tercero y cuarto habían cursado dos y tres años respectivamente, por lo que se puede estimar que se hallaban aún en una etapa inicial de su interlengua, teniendo en cuenta que el tiempo total dedicado a la materia Lengua Española era únicamente de cuatro horas semanales. En lo referente al proceso de aprendizaje, los alumnos se encontraban en una situación institucionalizada de asimilación del input proveniente del entorno escolar, es decir, que sus objetivos eran estrictamente académicos y orientados a la realización del examen final.

\section{Comentario y análisis de los resultados}

Ya hemos dicho que el proceso de análisis de las producciones idiosincrásicas permite determinar las causas, frecuencia y gravedad de cada una de ellas y determinar hasta qué punto es posible confirmar las hipótesis sobre los factores que generan mayor dificultad en el proceso de adquisición, así como la mayor o menor influencia ejercida por la lengua materna en dicho proceso. En cuanto al análisis de los errores propiamente dicho, describimos la interlengua de los informantes de manera sintética, presentando las producciones erróneas analizadas desde el punto de vista cuantitativo, mediante una serie de gráficos que indican numéricamente la aparición de los errores. Queremos recordar que los resultados serán mucho más provechosos si antes de pasar al estudio del corpus propiamente dicho realizamos un trabajo previo de contraste en ambos sistemas de las categorías lingüísticas implicadas en el análisis, ya que esta labor será muy útil para predecir áreas que van a ser en mayor medida objeto de error. No se trata de un examen pormenorizado de todos y cada uno de los usos de estos elementos y estructuras, sino que nos concentraremos en los casos en los que las dos lenguas presentan diferencias notables que pueden inducir al alumno a equivocarse.

\subsection{Criterio lingüístico}

Presentamos una primera clasificación de los errores por categorías, concretamente errores gráficos, morfológicos, sintácticos, léxicos y discursivos. Hemos considerado errores gráficos los problemas relacionados con la puntuación y las mayúsculas, el acento gráfico, la segmentación y unión de palabras, la alteración del orden de las letras, la confusión, omisión y adición de letras en una misma palabra, la confusión de grafemas para el mismo fonema y la apócope. Hemos clasificado como errores morfológicos, aquellos que atañen a las categorías del artículo, pronombres personales en función de sujeto y de complemento, pronombres reflexivos, demostrativos, posesivos, cuantificadores (numerales, indefinidos $\mathrm{e}$ indeterminados), todas las preposiciones, conjunciones coordinativas y subordinativas, relativos, adverbios, confusión de género y número de sustantivos y adjetivos y el uso de los tiempos verbales. Hemos dividido los errores sintácticos en dos grupos, por un lado, los que afectan a la estructura de la oración (orden de los elementos oracionales, omisión o adición de dichos elementos y falta de concordancia en género, número y de los tiempos verbales) $\mathrm{y}$, por otro lado, los errores relativos a la relación de coordinación y subordinación entre oraciones. Hemos considerado léxicos los errores de forma (como son el uso de un significante español próximo y las formaciones no atestiguadas en español creadas por derivación o composición) y de contenido (impropiedad semántica y de registro, pares de verbos no 
intercambiables, cambios de significado de ser y estar, perífrasis verbales, falsos amigos e interferencia de otras lenguas conocidas por los estudiantes). Los errores discursivos revelan casos de falta de coherencia interna del texto (relativa al contenido, organización de las ideas y adecuación de los conectores y marcas de cohesión textual) y determinan el grado de adecuación a la situación de comunicación.

Los más numerosos son los errores gráficos, con un total de 870 muestras, principalmente de acentuación (503 entre omisión, adición y confusión del acento gráfico) y de tipo ortográfico (omisión de los signos de exclamación e interrogación, 55 errores, y omisión, adición y confusión de grafemas en el interior del lexema, 226 errores) ${ }^{21}$.

Son 779 los errores relacionados con los elementos morfológicos de la oración, los más recurrentes atañen a las preposiciones, con 261 muestras, seguidas de los errores relacionados con el uso de los tiempos verbales, con 132 errores. También el artículo y los pronombres personales denotan algunos problemas, especialmente de adición, con 78 y 75 casos respectivamente. Otros elementos morfológicos resultan menos confusos para los estudiantes a juzgar por el número de producciones idiosincrásicas, es el caso de las conjunciones coordinativas y subordinativas ( 40 errores) y de los adverbios ( 55 errores), probablemente por la escasa frecuencia de uso de dichos elementos.

Los errores léxicos que aparecen en el corpus son 313, tanto de forma, con 59 casos de producción de vocablos inexistentes en español, como de contenido, los más profusos de confusión entre ser y estar (42 casos) y entre pares de verbos no intercambiables, como estar/haber impersonal, ir/venir, poner/quitar, etc. (45 casos). Son 85 los errores léxicos provocados por interferencia de la lengua materna y de otras lenguas conocidas por los informantes, aunque entre estas muestras aparecen muchos casos evidentes de permutación directa por el término en italiano, cuya única explicación es el desconocimiento de la palabra en español y cuya presencia nada nos dice sobre la interlengua de los estudiantes.

En cuanto a los errores sintácticos, son los menos abundantes, 187 casos que conciernen, sobre todo, a las oraciones coordinadas, de los cuales 36 son de discordancia en número de los elementos del sintagma nominal y 29 en persona gramatical en el sintagma verbal, además de algunos fallos de orden, omisión y uso redundante de los elementos que forman parte de la estructura de la oración $(19,29$ y 14 respectivamente).

Por último, por lo que se refiere a los errores discursivos, no hay que olvidar que muchos de los errores gráficos, morfológicos, léxicos y sintácticos interfieren también en la valoración de la competencia discursiva de los informantes, no obstante, hemos preferido no incluirlos numéricamente, por ser difícilmente computables. Presento un gráfico en cifras absolutas de la distribución de los errores según la categoría a la que atañen.

21 El recuento de los errores da un total de 2.149 , repartidos por todo el corpus, lo cual supone una media de 48,8 por enunciado, aunque parece un valor excesivo, hay que considerar que los errores más reiterados son de tipo gráfico, y en especial los casos de omisión del acento, es decir, que son errores muy numerosos pero de escasa relevancia para la transmisión del mensaje. 


\section{DISTRIBUCIÓN DE LOS ERRORES POR CATEGORIAS}

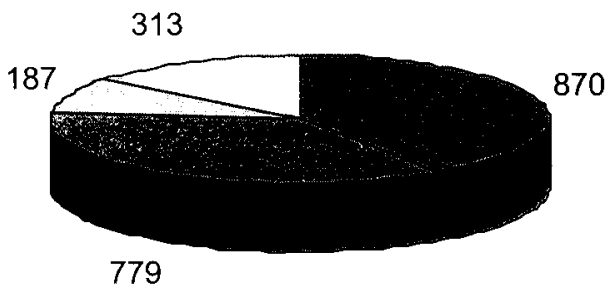

\begin{tabular}{l} 
- gráficos \\
- morfológicos \\
- léxintácticos \\
\hline
\end{tabular}

\subsubsection{Distribución de los errores por cursos}

Si se distribuyen por cursos los errores hallados, 1516 errores, o lo que es lo mismo, el $70 \%$, conciernen a enunciados de estudiantes del segundo año, 270 errores son de alumnos del tercer año, concretamente el $13 \%$, y 363 errores son de estudiantes del cuarto año, o sea, el $17 \%$. Aunque la mayor parte desaparecen completamente en el nivel intermedio, los errores cometidos por los estudiantes del segundo año son numerosos y engloban prácticamente todos los sectores de la lengua, lo que demuestra la inestabilidad de su interlengua. Los errores de los estudiantes del tercer y cuarto año son también considerables, pero de menor importancia, son casos de confusión de algunas áreas lingüísticas que no presentan grandes riesgos de fosilización y que no interfieren en la consecución de la comunicación.

En general, podemos afirmar que todos los textos revelan gran inseguridad en el empleo de las reglas básicas de ortografía y acentuación. En cuanto al léxico, parecen tener menos dudas, ya que en general los estudiantes se expresan con propiedad y riqueza formal. Algunos ejemplos de error recurrente con relación a la morfología del español es el uso de expresiones perifrásticas italianas en lugar del neutro lo, la omisión del reflexivo por hipercorrección y la confusión del pronombre demostrativo esto en lugar de este; en cuanto a las preposiciones, es característica la omisión de la preposición $a$ ante complemento directo de persona, la adición de la preposición de (en la estructura de+infinitivo en lugar de $q u e+$ subjuntivo) y su omisión en otros contextos y la elección errónea de las preposiciones por y para; otros errores se relacionan con las conjunciones, por ser una categoría gramatical de difícil adquisición y por su gran frecuencia de uso. En el ámbito de la sintaxis, en cambio, es notable la falta de concordancia en género y número del grupo nominal, y todos aquellos errores que provienen del uso, en los niveles avanzados, de una estructura sintáctica más compleja. En definitiva, la gran mayoría de los errores que aparecen en el corpus se originan en el nivel elemental, disminuyendo notablemente en niveles superiores. En el siguiente gráfico se puede observar la distribución de los errores por cursos: 


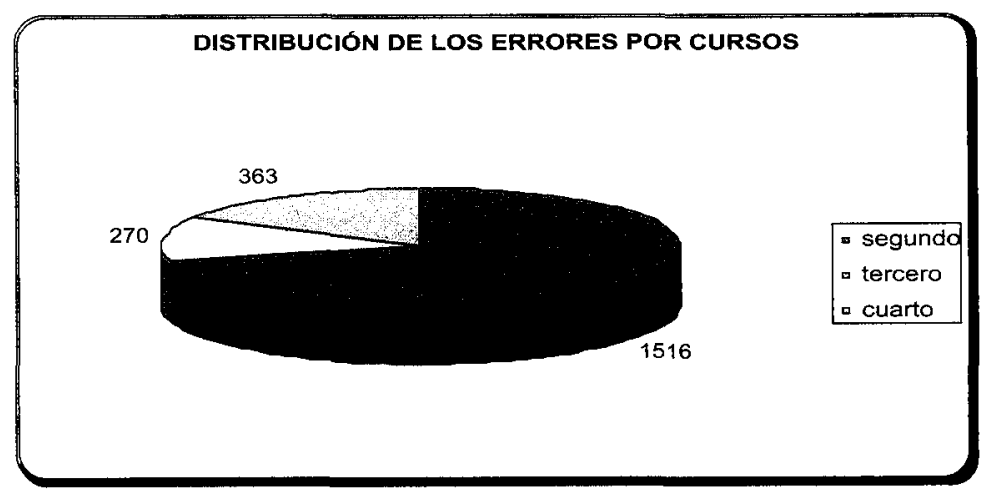

\subsubsection{Distribución de los errores por tipos}

En cuanto a la clasificación de las producciones idiosincrásicas según el criterio lingüístico, el orden o colocación de los varios componentes oracionales no implica grandes dificultades para los estudiantes italianos. La mayor inestabilidad surge en la selección de formas, el $52 \%$ son casos de elección errónea ( 1.101 errores), frente a un número menor de casos de omisión, el 33\% (716 errores), adición, el 14\% (303 errores), y colocación falsa, sólo el $1 \%$ (29 errores).

Relacionando esta clasificación con la división por categorías se observa, en primer lugar, que los casos de elección errónea prevalecen en los sectores léxico y morfológico, como era de prever, en segundo lugar, los pocos ejemplos de falsa colocación identificados conciernen a errores sintácticos de orden incorrecto de los elementos de la oración, en tercer lugar, la omisión se da principalmente en el uso de elementos gráficos — sobre todo de la acentuación-y en menor medida de elementos morfológicos y, por último, el porcentaje de adiciones se reparte entre dos categorías, errores gráficos y morfológicos. Véase el gráfico con la distribución de los errores según el criterio lingüístico.

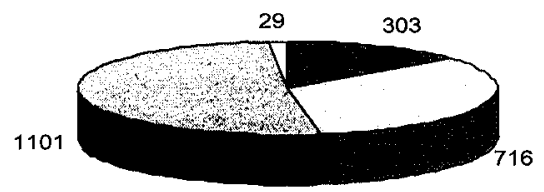




\subsection{Criterio etiológico}

Teniendo en cuenta el criterio etiológico de clasificación, hemos querido determinar el grado de protagonismo de la L1 como factor causante de los errores y en algunos casos de su persistencia. La mayor parte de los errores es de tipo interlingual, frente a un número reducido de errores producidos por desconocimiento de los rasgos de la lengua objeto. Pero no todos los errores se deben a fenómenos de transferencia, los errores intralinguales encontrados han sido provocados por varios factores, por ejemplo, el uso incorrecto de algunas excepciones de las reglas de la L2 ya estudiadas, hipercorrección por inestabilidad ante categorias lingüísticas dificiles, confusión con otras estructuras gramaticales o hipergeneralización de reglas.

Los casos de transferencia lingüística son muchos y variados, el análisis de las muestras evidencia la presencia de estructuras gramaticales y vocablos que son el resultado de la interferencia de la lengua materna del informante ${ }^{22}$. Como sabemos, cuando se trata de lenguas afines, la lengua materna influye de forma activa en la adquisición de la L2 como conocimiento preexistente al que el estudiante acude para superar ciertas carencias, así, en los primeros estadios de interlengua es normal que la influencia de la lengua materna sea mayor, ya que se conocen menos las oposiciones propias del español, sobre todo cuando la L2 presenta un elemento muy marcado, recurriendo a la estructura equivalente de la L1 si ésta es más simple. Las áreas más problemáticas que encuentra un estudiante italiano son, principalmente, la presencia en español de una oposición funcional que en su lengua no existe, la falta de correspondencia entre una regla de su lengua materna y de la lengua objeto y la existencia de una misma categoría en ambas lenguas pero con usos invertidos.

\subsection{Criterio comunicativo}

Según el criterio comunicativo son pocos los errores que dificultan la comunicación (exceptuando algunos fragmentos que contienen impropiedades semánticas y ejemplos de formación de palabras no atestiguadas en español), especialmente en los enunciados de los grupos de los cursos avanzados, aunque la recurrencia a veces produce irritación.

\subsection{Criterio pedagógico}

Por último, según el criterio pedagógico, los errores son transitorios en la mayor parte de los casos frente a un reducido número de errores fosilizables y una cantidad aún menor de errores permanentes o fosilizados. La mayor parte de los errores que conciernen a la producción de léxico, a la capacidad discursiva de los estudiantes y a las confusiones provocadas

22 Uno de los fenómenos que llama la atención y en è que más queremos insistir, es la influencia de las estructuras de la lengua materna en el proceso de aprendizaje del español por italianos y, por ello, en la formación de su interlengua. Este fenómeno puede ser también un proceso activo, una estrategia de tipo cognitivo, cuando la lengua materna viene utilizada como referente para formular una serie de hipótesis sobre la L2, de hecho, en ocasiones la transferencia es positiva — ya que el resultado es una producción que se ajusta a las reglas de la lengua meta-, pero en la mayoría de los ejemplos es negativa, pues da lugar a una estructura incorrecta o inaceptable. En la enseñanza institucionalizada puede ser interesante potenciar estas estrategias cognitivas, pero siempre vigilando que no se produzcan fosilizaciones, sobre todo en los casos de evidente similitud interlingüistica. 
por distracción, son individuales. Son muy numerosos, en cambio, los errores colectivos, sobre todo cuando atañen a categorías morfológicas — como las preposiciones- y ortográficas - como la acentuación-, además, presentan mayor riesgo de fosilización y suelen ser bastante característicos de los hablantes italianos.

\subsection{Evolución de los errores}

Otra perspectiva de análisis del corpus es el estudio de la evolución de los errores en los tres cursos, para verificar si el mejoramiento es global y paralelo en todos los estudiantes y qué desviaciones oponen mayor resistencia para su superación. Como ejemplo de errores gráficos que se mantienen en cursos avanzados, los problemas de acentuación y de confusión de grafemas son los más representativos, frente a los casos de uso de mayúsculas o de apóstrofe, que parecen solventarse ya en los primeros estadios de competencia lingüística. Los errores morfológicos que permanecen son la adición del artículo, el uso de los pronombres átonos en general, la omisión del pronombre reflexivo, la elección del indefinido y el uso de buena parte de las preposiciones, mientras que hay errores que desaparecen casi completamente, como la adición del pronombre en función de sujeto o el uso del demostrativo neutro en lugar del masculino singular. En cuanto a la sintaxis, en los niveles más avanzados desaparecen los casos de orden sintáctico incorrecto, mientras que se mantienen muchos ejemplos de omisión de elementos de la oración, en cambio, el tipo de error que presenta una evolución menor es, sobre todo, la concordancia en número y, en menor medida, en persona gramatical. Los errores léxicos disminuyen bastante en los textos de tercero y cuarto curso (los errores de paronimia y de interferencia del italiano y de otras lenguas desaparecen casi totalmente), exceptuando casos de impropiedad semántica y perífrasis verbales, fenómeno explicable por la mayor madurez lingüística de estos estudiantes. Por último, en cuanto a la capacidad discursiva, como es lógico, se expresan con más propiedad los estudiantes de los cursos más avanzados.

\subsection{Características del corpus}

He examinado las características del corpus, es decir, la extensión de los textos calculado según la media de oraciones por enunciado y la media de palabras por texto para determinar si aumenta o disminuye la consistencia de los enunciados proporcionalmente al curso al que pertenecen, y si los errores disminuyen, se mantienen o si crecen con el aumento de la producción textual.

Como se puede observar en los gráficos que presentamos, la competencia lingüística y discursiva de los estudiantes mejora proporcionalmente al tiempo dedicado al aprendizaje de la lengua, ya que aumenta la producción y disminuyen los errores. Por lo que respecta a la extensión de los textos, presentan una media creciente que aumenta progresivamente en los tres cursos, ya que los textos de segundo revelan una media de 16,7 líneas por enunciado, los de tercero de 21,8 y los de cuarto de 26,5. El número de palabras que integran el corpus es de 10.370, también la media por texto sigue una progresión por cursos: 208,9 es la media de palabras de las composiciones de segundo, 269 de los textos de tercero y 333 de los de cuarto. Existen varias desviaciones de la media, textos de gran longitud o de pocas líneas de extensión, especialmente en el primer nivel. Por otra parte, el número de frases por texto 

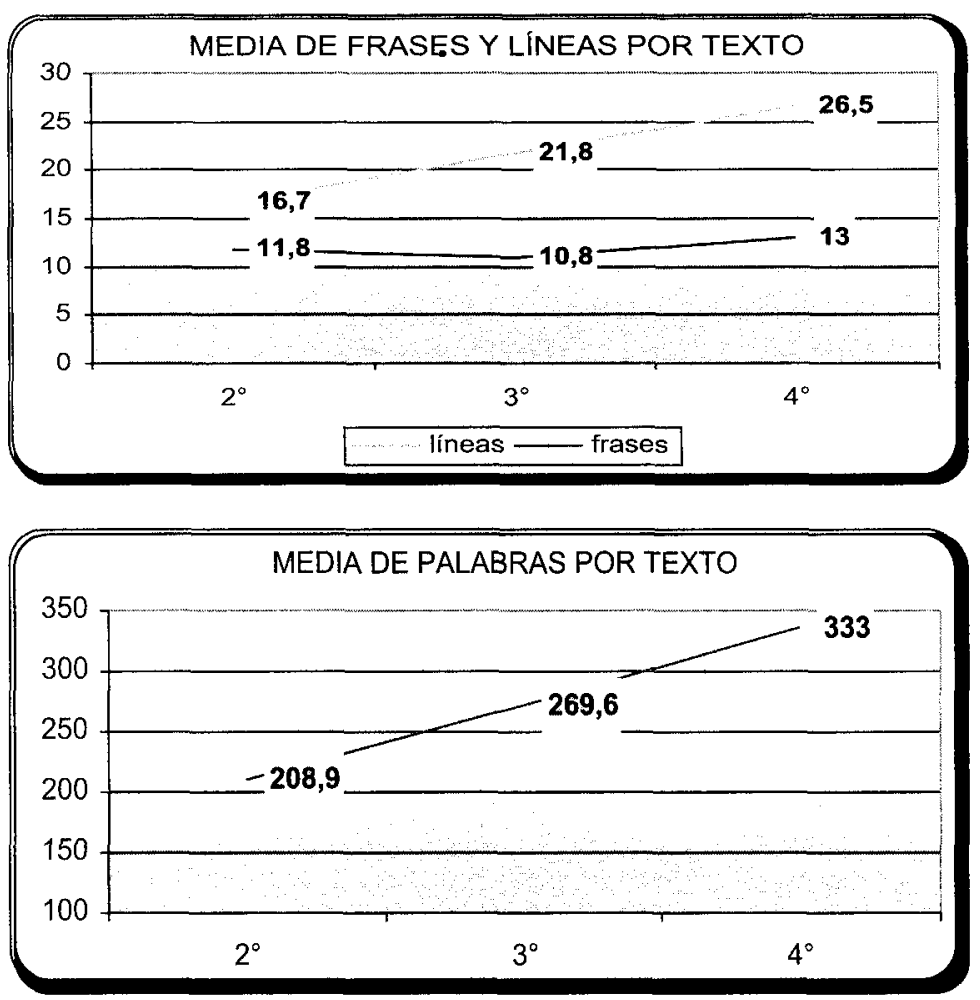

es también un buen indicador de la extensión y elaboración de los enunciados, de hecho, se produce un gran salto en los textos de los estudiantes del cuarto curso, con una media de 13 frases por enunciado.

\subsection{Cuestionario sobre su propia concepción de los errores}

Durante los primeros minutos de nuestro encuentro con los estudiantes, con motivo de la elaboración de las pruebas y la recogida del corpus, se les pidió que respondieran un breve cuestionario. En la primera parte del mismo, titulado Cuestionario sobre tu experiencia personal, se pedían una serie de informaciones relacionadas con su propia experiencia como estudiantes de españo ${ }^{23}$, en cambio, la segunda parte del sondeo se titulaba Cuestionario sobre tu propia concepción de los errores, y consistía en una reflexión personal de los estudiantes sobre su propio proceso de aprendizaje y en especial sobre las dificultades con las que se

23 ¿Durante cuántos años has estudiado español? (menos de uno; entre uno y cinco; entre cinco y diez; más de diez). ¿Has estado alguna vez en un pais hispanohablante? (no, nunca; si, como turista; si, como estudiante; sí, trabajando), ¿Cuánto tiempo duró tu estancia? (pocos dias; un mes; varios meses, varios años), ¿Cómo ha sido tu formación? (estudié español en la universidad o en otro tipo de escuela italiana; he realizado cursos en España o Hispanoamérica). 
suelen enfrentar en dicho proceso ${ }^{24}$. Analizando tanto los textos como los resultados de la investigación, pudimos comprobar que muchos alumnos manifestaban carencias de tipo lingüístico y pragmático por no haber vivido situaciones de inmersión lingüística y haber estudiado español únicamente en un entorno académico. Muchos errores provenían de esta falta de exposición del alumno a situaciones de intercambios comunicativos reales en los que poner en práctica de manera contextualizada los conocimientos adquiridos en el aula. De hecho, en el momento de la recogida del cuestionario, el $86 \%$ de los informantes había cursado estudios de español exclusivamente en su país y la gran mayoría, el $73 \%$, no había estado nunca en un país hispanohablante, ni siquiera por motivos laborales o de ocio. El porcentaje de alumnos que había estado alguna vez en un país hispanohablante era del 14\%, pero sólo menos de la mitad había completado su formación realizando cursos en algún país de habla hispana, de hecho, para la mayor parte de ellos, su estancia fue de pocos días o como mucho de un mes, y sólo una persona pasó más tiempo en un país hispanohablante, su permanencia fue de diez meses. En cuanto a la duración de los estudios, la mayoría de los estudiantes, el $95 \%$, había estudiado español durante un periodo de entre uno y cinco años, el $5 \%$ durante menos de un año y ninguno de ellos durante más de cinco años.

Analizando las respuestas obtenidas en la segunda parte del cuestionario propuesto a los estudiantes, observamos que, en general, eran bastante homogéneas. Los resultados son muy interesantes, ya que denotan que nuestros estudiantes son conscientes de que muchos de sus errores están motivados por la interferencia de su lengua materna y por las similitudes y diferencias que ambos sistemas lingüísticos presentan, además, son capaces de determinar qué sectores perciben como inestables en su proceso de aprendizaje, lo cual puede ser de gran utilidad para el profesor, para insistir especialmente en esas áreas problemáticas. Respecto a la primera pregunta, todos los estudiantes afirmaron que cometían errores y, en la mayor parte de los casos con mucha frecuencia, excepto algunos estudiantes del cuarto año que no los consideraban excesivos aunque, en general, no sabían con exactitud la asiduidad ni el porqué de su aparición. En cuanto a la segunda pregunta, mencionaron como errores más corrientes la acentuación y varias cuestiones más puramente gramaticales, casi todos parecían coincidir en el uso de algunos verbos - hay quien afinó más y citó el uso de ser y estar - y, en general, las preposiciones, y en muchas ocasiones se habló de errores relacionados con el léxico. Por último, a la tercera pregunta sobre si sus errores son similares a los de los demás estudiantes italianos, todos respondieron afirmativamente. Es curioso, porque para algunos alumnos el motivo era, principalmente, la semejanza entre su lengua materna y el español, aunque hubo quienes atribuyeron la responsabilidad de las confusiones al hecho contrario, a las diferencias entre ambas lenguas y, por último, hay quien opina que el origen de los errores es la falta de estudio y la imposibilidad de viajar a países donde poder practicar la lengua. Es interesante observar que ninguno de los informantes aludió a los errores resultantes de la comunicación oral, tal vez porque en muchas programaciones didácticas de los cursos impartidos en entornos institucionales este tipo de ejercitación es casi inexistente, dando prioridad a la adquisición de conocimientos de tipo gramatical.

24 ¿Crees que cometes errores?, ¿con qué frecuencia?; ¿Qué tipo de errores son?, ¿cuáles son los más corrientes?; Basándote en tu experiencia personal, ¿crees que los errores que tú cometes son similares a los de otros estudiantes de español (italianos)? Si la respuesta es afirmativa, ¿cuál piensas que será el motivo de este fenómeno? 


\section{Conclusiones}

Para concluir, solo decir que con los resultados de este trabajo hemos querido demostrar que el proceso de aprendizaje del español como lengua extranjera es muy similar cuando los estudiantes comparten la misma lengua materna y, con ello, que el desarrollo de la interlengua de estos estudiantes es paralelo, que existen errores característicos de los estudiantes italianos, que utilizan el mismo tipo de estrategias y que la interferencia de la lengua materna es causa continua de error, entre otras cosas. Creemos que mediante este tipo de estudios se pueden identificar los errores más recurrentes y las áreas de mayor dificultad, se puede intervenir en la transmisión de estrategias, en la programación de cursos y en la preparación de material didáctico específico, además de llegar a conclusiones que no obtenemos mediante la simple práctica docente, ya que los datos nos muestran no sólo el tipo de error que produce el estudiante, sino también el contexto en el que dicho error aparece, la transitoriedad o el posible riesgo de fosilización y su evolución en los diferentes niveles de competencia lingüística. Estamos convencidos de la importancia de los estudios comparativos entre lenguas afines, pues en muchas ocasiones una buena labor de reflexión y de análisis contrastivo entre la lengua materna de los estudiantes y la lengua objeto puede evitarnos muchos problemas, tanto a nosotros profesores como a los estudiantes que se enfrentan a ese gran reto que es el aprendizaje de segundas lenguas. Quiero resaltar una vez más la importancia que entraña la aplicación directa en el aula, pues si tenemos en cuenta los resultados de nuestras investigaciones en el momento de programar nuestros cursos de español, evitaremos derrochar nuestras energías y las de nuestros estudiantes proponiendo explicaciones ineficaces. Confiamos en que este artículo servirá de estímulo para la realización de nuevos estudios contrastivos que impulsen la formación de una sólida base metodológica tan necesaria actualmente en el ámbito de adquisición de lenguas por grupos homogéneos.

\section{Referencias bibliográficas}

Adjémian, C. y J. M. Liceras (1984): "Accounting for Adult Acquisition of Relative Clauses: Universal Grammar, L1 and Structuring the Intake". En Eckman, F., L. Bell y D. Nelson (eds.): Universals of Second Language Acquisition. Rowley, Mass., Newbury House.

Alarcos Llorach, E. (1995): Gramática de la lengua española. Madrid, Real Academia Española, Espasa-Calpe.

Alcaraz Varó, E. y M. A. Martínez Linares (1997): Diccionario de lingüística moderna. Barcelona, Ariel.

Alvar Ezquerra, M. (dir.) (2000): Introducción a la lingüistica española. Barcelona, Ariel Lingüistica.

Álvarez Martínez, M. A. (1989): El pronombre I. Madrid, Arco/Libros.

Aragona, A. (1992): Diccionario de ambigüedades: español-italiano, italiano-español. Messina, EDAS.

Arribas, G. y E. Landone (2003): Dificultades del español para hablantes de italiano. Madrid, Prácticos SM.

Baralo, M. (1994): Errores y fosilización. Madrid, Edinumen.

Baralo, M. (1999): La adquisición del español como lengua extranjera. Madrid, Arco/Libros. Battaglia, G. (1989): Gramática italiana (para estudiantes de habla española). Roma, Baracci. Bellini, G. (1994): Grammatica della lingua spagnola. Milano, LED. 
Betti, S. (2002): "Uso de algunas palabras extranjeras y neologismos en el italiano y en el español de hoy. Una propuesta metodológica”. En Cuadernos Cervantes, núm. 39, Alcalá de Henares, págs. 26-32.

Bini, M. y P. Guil (2002): "Italiano y español: algunos puntos de contraste". En Carabela, núm. 51, Madrid, SGEL.

Blanco Picado, A. I. (2002): "El error en el proceso de aprendizaje". En Cuadernos Cervantes, núm. 38, Alcalá de Henares.

Caballero Rubio, M. C. y J. B. Corral Hernández (1997): "Estudio contrastivo de las preposiciones locativas en español e italiano". En Frecuencia ELE, núm. 5, Alcalá de Henares, págs. 42-49.

Calvi, M. V. (1981): "Interferenze delle altre lingue straniere studiate nell'apprendimento dello spagnolo". En A.A.V.V., Didattica della lingua e lingue iberiche, Atti del Convengo dell'Associazione Ispanisti Italiani, L'Aquila, págs. 9-27.

Calvi, M. V. (1995): Didattica di lingue affini. Spagnolo e italiano. Milano, Guerini Scientifica.

Calvi, M. V. (2004): "Aprendizaje de lenguas afines: español e italiano", redELE 1: http://formespa. rediris.es/revista.

Calvi, M. V. y Monti, S. (1991): Nuevas palabras-Parole nuove, dizionario spagnolo-italiano e italiano-spagnolo di neologismi e di espressioni colloquiali. Torino, Paravia.

Calvo Rigual, C. y A. Giordano (1997): Diccionario italiano-español e español-italiano. Barcelona, Herder.

Carrera Díaz, M. (2002): Grammatica spagnola. Bari, Editori Laterza.

Castañeda Castro, A. y V. Peña Sánchez (1995): "Adquisición del subjuntivo y nivel de dominio en estudiantes italianos de español como lengua extranjera". En Aspectos de la enseñanza de lenguas extranjeras, Granada, Universidad de Granada, págs. 133-142.

Ciliberti, A. (1994): Manuale di glottodidattica. Firenze, La Nuova Italia.

Corder, S. P. (1967): "The Significance of Learners Errors". En International Review of Applied Linguistics, vol. V, 4.

Corder, S. P. (1981): Error Analisis and Interlenguage. Oxford, University Press.

De Kock, J. (1996): "Este, ese y aquel en traducción alemana, francesa, inglesa e italiana". En Gramática española, enseñanza e investigación. Apuntes metodológicos: Lingiïstica contrastiva, Salamanca, Ediciones de la Universidad de Salamanca, págs. 13-43.

De La Torre, S. (1993): Aprender de los errores. El tratamiento didáctico de los errores como estrategia de innovación. Madrid, Escuela española.

Fernández López, S. (1989): "Corregir y evaluar desde la perspectiva comunicativa", Actas de las Primeras Jornadas Pedagógicas de ASELE, Granada, Universidad de Granada, págs. 13-29.

Fernández López, S. (1994): "Problemas discursivos en la interlengua de aprendices de español como lengua extranjera". En Montesa, S. y A. Garrido Moraga: Actas del II Congreso Nacional de ASELE, Español para extranjeros: didáctica e investigación. Málaga, págs. 267-279.

Fernández López, S. (1997): Interlengua y análisis de errores en el aprendizaje de español como lengua extranjera. Madrid, Edelsa.

Fernández, M. C. (1999): Las preposiciones en español. Valores y usos. Construcciones preposicionales. Salamanca, Ediciones Colegio de España.

Finasi Parolo, M. y G. Giovannetti Muñoz (1998): Nueva gramática de español para italianos. Torino, Petrini Editore.

Fries, C. (1945): Teaching and Learning English as a Foreign Language. Ann Arbor, University of Michigan Press.

Galán Bobadilla, A. (1996): "Análisis contrastivo y análisis de errores en el aula de español como lengua extranjera". En S. Montesa Peydró y P. Gomis Blanco (eds.): Tendencias actuales en la enseñanza del español como lengua extranjera. I, Actas del Quinto Congreso Internacional de ASELE, Santander, págs. 10-109. 
Gil, J. (1994): "Problemas que presenta la enseñanza del español a italianos". En Montesa, S. y A. Garrido Moraga: Actas del II Congreso Nacional de ASELE, Español para extranjeros, didáctica e investigación. Málaga, págs. 117-124.

Gómez Torrego, L. (1998): Gramática didáctica del español. Madrid, SM.

González Royo, C. (1986): "Italiano y español: análisis de los errores gramaticales en la lengua escrita durante el aprendizaje del español por italófonos". En Actas del Tercer Congreso Nacional AESLA, págs. 541-556.

Gorris Yagüe, I. (2003): Manual de español para italianos. Bolonia, Petrarca.

Gutiérrez Quintana, E. (2004a): "El subjuntivo en español e italiano. Propuesta didáctica". En Actas del VIII Congreso Nacional de Lingüística y Filología, Rio de Janeiro.

Gutiérrez Quintana, E. (2004b): "Los pronombres personales en español un área problemática para los estudiantes italianos". En Actas del V Congreso Andaluz de Lingüística, Granada.

Gutiérrez Quintana, E. (2004c): "Un posible modelo de análisis contrastivo entre lenguas afines". En Actas del I Simposio de Didáctica del Español para Extranjeros. Teoria y Práctica, Instituto Cervantes de Rio de Janeiro.

Gutiérrez Quintana, E. (2004d): Aspectos gramaticales contrastivos en español e italiano. Alcalá de Henares, Tesis doctoral defendida en la Universidad de Alcalá.

Gutiérrez Quintana, E. (2005a): "Análisis de errores en la producción escrita de italianos aprendices de E/LE", REALE, Universidad de Alcalá.

Gutiérrez Quintana, E. (2005b): "Aproximación contrastiva a la categoría del artículo en español, italiano y portugués". En Actas del IV Congreso Internacional de Lingüística Contrastiva (ICLC-4), Santiago de Compostela.

Lado, R. (1957): Linguistics Across Cultures. Ann Arbr, University of Michigan Press.

Larsen-Freeman, D. y M. H. Long (1994) (versión española de Molina Martos, I. y P. Benítez Pérez): Introducción al estudio de la adquisición de segundas lenguas. Madrid, Gredos manuales.

Liceras, J. M. (1992): La adquisición de las lenguas extranjeras. Madrid, Visor.

López Valverde, M. D. (2002): "La fosilización de algunos errores de los alumnos de lengua materna italiana", Frecuencia-L, núm. 19, Madrid, Edinumen, págs. 11-15.

Luque Toro, L. (1994): "Problemas de lingüística contrastiva en la enseñanza de español para extranjeros (nivel superior)". En Montesa, S. y Garrido Moraga, A.: Actas del II Congreso Nacional de ASELE, Español para extranjeros: didáctica e investigación. Málaga, págs. 125-133.

Martínez Amador, E. (1988): Diccionario italianolespañol, español/italiano. Barcelona, Sopena.

Matte Bon, F. (2001): Gramática comunicativa del español. Tomo I, Madrid, Edelsa.

Matte Bon, F. (2004): "Análisis de la lengua y enseñanza del español en Italia", redELE, núm, 0: http://formespa.rediris.es/revista.

Montesi, M. (2000): "El punto de vista de un hablante italiano", Cuadernos Cervantes, núm. 29, Universidad de Alcalá de Henares, págs. 34-40.

Penadés Martínez, 1. (coord.) (1999): Lingüistica contrastiva y análisis de errores (español-portugués y español-chino). Madrid, Edinumen, págs. 7-19.

Ribas Moliné, R. y A. D'aquino Hilt (2004): ¿Cómo corregir errores y no equivocarse en el intento? Madrid, Edelsa.

Sañé, S. (1992): Falsos amigos al acecho: Dizionario di false analogie tra spagnolo e italiano. Bologna, Zanichelli.

Santos Gargallo, I. (1993): Análisis Contrastivo, Análisis de Errores e Interlengua en el marco de la Linguïística Contrastiva. Madrid, Síntesis.

Santos Gargallo, I. (1994): "Análisis de errores: valoración gramatical y comunicativa en la expresión escrita de estudiantes de ELE. Una propuesta didáctica". En Montesa, S. y A. Garrido Moraga: Actas del II Congreso Nacional de ASELE, Español para extranjeros: didáctica e investigación. Málaga, págs. 169-174. 
Santos Gargallo, I. (1999): Lingüística Aplicada en la enseñanza-aprendizaje del español como lengua extranjera. Madrid, Arco/Libros.

Selinker, L. (1992): Rediscovering Interlanguage. Londres, Longman.

Tam, L. (1993): Dizionario spagnolo-italiano, italiano-spagnolo. Novara, De Agostini.

Torijano Pérez, J. A. (2004): Errores de aprendizaje, aprendizaje de los errores. Madrid, Arco/Libros.

Vázquez, G. (1987): "Hacia una valoración positiva del concepto de error". En Actas de las Jornadas de Didáctica del Español como Lengua Extranjera. Madrid, Ministerio de Cultura, págs. 151163.

Vázquez, G. (1991): Análisis de errores y aprendizaje de español/lengua extranjera. Frankfurt Am Main, Peter Lang.

Vázquez, G. (1992): "El concepto de error: estado de la cuestión y posibles investigaciones". En Actas del Congreso de Lengua Española. Madrid, Instituto Cervantes, págs. 472-496.

Vázquez, G. (1998): ¿Errores? ¡Sin falta! Madrid, Edelsa.

Wardhaugh, R. (1970): "The Contrastive Analysis Hypothesis", TESOL Quartely 4, págs. 123-136. 